\title{
The Power of the Blue Tick ( ): Ugandans' experiences and engagement on Twitter at the onset of the COVID-19 pandemic
}

\author{
Lynn Kirabo* \\ lkirabo@andrew.cmu.edu \\ Carnegie Mellon University \\ Pittsburgh, PA, United States
}

\author{
Moses Namara* \\ mosesn@g.clemson.edu \\ Clemson University \\ Clemson, SC, United States
}

\author{
Nathan McNeese \\ mcneese@clemson.edu \\ Clemson University \\ Clemson, SC, United States
}

\begin{abstract}
To raise awareness and communicate measures aimed at curbing the ongoing COVID-19 pandemic, the Ugandan government used a combination of traditional and social media platforms. This included nationally televised presidential addresses on early coping efforts. We examine the impact of these communications by using public Twitter data to explore Ugandans' experiences, conversations, and engagement leading up to, during, and after these addresses. We found that tweets from verified accounts received more user engagement than tweets from non-verified accounts. We also used a Louvian clustering algorithm to identify the topics around which tweet clusters occurred. Some topics were unique to Uganda's COVID-19 response, such as lockdown and truck drivers. Thematic analyses within the five clusters uncovered sub-themes on conversation dynamics, and Twitter use during the pandemic. Overall, we use Uganda as a case example of using social media in a crisis (such as a pandemic) to provide insight into the African experience.
\end{abstract}

\section{CCS CONCEPTS}

- Human-centered computing $\rightarrow$ Social network analysis; • Social and professional topics $\rightarrow$ Geographic characteristics.

\section{KEYWORDS}

computer-mediated communication, crisis informatics, pandemic response, risk communication, social media, Uganda, Kampala, Twitter

\section{ACM Reference Format:}

Lynn Kirabo, Moses Namara, and Nathan McNeese. 2021. The Power of the Blue Tick ( Ugandans' experiences and engagement on Twitter at the onset of the COVID-19 pandemic. In 3rd African Human-Computer Interaction Conference (AfriCHI 2021), March 8-12, 2021, Maputo, Mozambique. ACM, New York, NY, USA, 10 pages. https://doi.org/10.1145/3448696.3448746

\section{INTRODUCTION}

The novel coronavirus disease 2019 (COVID-19), declared by the World Health Organization as a pandemic on 11 March 2020 [42],

"These authors contributed equally to this research.

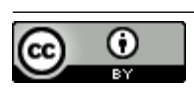

This work is licensed under a Creative Commons Attribution International 4.0 License.

AfriCHI 2021, March 8-12, 2021, Maputo, Mozambique

(C) 2021 Copyright held by the owner/author(s)

ACM ISBN 978-1-4503-8869-6/21/03

https://doi.org/10.1145/3448696.3448746 drastically changed people's lives and ways of living. To mitigate its spread, various governments implemented lockdown, and stayat-home directives that restricted the physical mobility and socialization of people $[22,30,35]$. Subsequently, most people's work and social lives shifted online (primarily for those with Internet access and/or whose work could be done remotely), leading to a drastic increase in the use of technology to communicate, stay informed and connect with others. As a result, social media platforms, such as Twitter, emerged as convenient channels to raise awareness and sources of information about the pandemic [26]. More specifically, Twitter was an essential tool in the identification of primary issues and concerns that the public had about the pandemic, served as an important communication link between the public, government, and health authorities, and was instrumental in the transmission of information and updates on the day-to-day progression rates of the pandemic $[6,16]$. However, Twitters' unique usage patterns and purposes during a crisis (e.g., a pandemic) remain largely unknown within the African context.

In this paper, we leverage public Twitter data to explore Ugandans' discourse, experiences, and engagement with information about the pandemic to offer insight on the unique ways in which Twitter was used during the pandemic from an African perspective. Ugandan government officials and institutions, such as the President ${ }^{1}$ and the Ministry of Health ${ }^{2}$, used Twitter to share and provide timely updates on the progression of the pandemic within the country. The citizenry also used Twitter to engage, document and share their personal experiences, thoughts, feelings during the pandemic. However, the heuristics that influenced their engagement (defined in this paper as the total number of times a user interacted with a tweet through retweets [37]) and the topics/concerns about the COVID-19 pandemic they shared on the platform remain unknown.

The main objectives of this study were two-fold: 1) to examine the discourse and engagement patterns of Ugandans on Twitter, and 2) to examine and identify the prevalent topics or conversations that people had or were interested in at the onset of the pandemic. Specifically, we examine the following two research questions:

- RQ1: What heuristics influenced user engagement (e.g., number of retweets) with tweets shared on Twitter?

- RQ2: What were the prominent topics and/or conversations on Twitter at the onset of the pandemic in Uganda?

To answer these research questions, we focused on public tweets shared before, during, and after the Ugandan president's 15 nationally televised presidential addresses between March 18th and

\footnotetext{
${ }^{1}$ https://twitter.com/KagutaMuseveni

${ }^{2}$ https://twitter.com/MinofHealthUG
} 
June 1st 2020. These addresses were meant to inform, boost morale, and update people on the government's efforts in mitigating the spread of COVID-19, with the excerpts disseminated and widely shared on Twitter. We find that majority of the tweets at the onset of the COVID-19 pandemic lockdown in Uganda were about the following topics: 1) the spread and progression of coronavirus, 2) the first COVID-19 case in Uganda, 3) the presidential addresses, 4) the lockdown directives (i.e., stay-at-home orders), and 5) the prevalent cases among truck drivers in Uganda who were granted permission to continue operations during the lockdown. We also observe that there was more user engagement with tweets from verified accounts than unverified accounts. These findings, topics and subsequent conversations provide insight into the types of accounts users are more likely to interact with and the specific usage of Twitter as a communication, information, and support channel during the pandemic in Uganda.

This study contributes to the AfriCHI research community, as well as crisis communication scholarship, in two ways: 1) we provide insight into the usage of technology (i.e., social media) by the online Ugandan public, focusing on their engagement patterns and conversation dynamics during the COVID-19 pandemic; and 2) we examine the importance of specific social media heuristics (e.g., account verification and number of followers) in the transmission of information within this context.

In the following sections, we discuss the related work regarding the use of social media in crises. In section 3 , we discuss our mixed analysis methodology. In section 4 , we present our findings, and the implications are discussed in Section 5. Section 6 conveys our limitations, followed by our conclusion.

\section{RELATED WORK}

Over the last decade, social media platforms have emerged as effective communication tools. As a result, researchers have devoted time and resources into investigating the information consumption habits of social media users [5, 9, 10, 14, 40, 43]. In this section, we present literature examining the role of social media during crises. We narrow the scope of our focus to environmental disasters and disease outbreaks, as these topical events are most closely aligned with the current pandemic. We end the section by examining prior work on heuristics that influence user engagement on Twitter.

\subsection{Social Media use during natural and environmental disasters}

During natural and environmental disasters (e.g., hurricanes, floods, and oil spills), Twitter users use the platform to report, share and collectively make sense of information. Users are specific about the information they share and the motivations of why they do so. For instance, Vieweg and Starbird [40] learnt that users were motivated to share information that they felt was relevant to individuals who were local to the event. These users had greater need for the information, specifically to aid in preparations and/or evacuations. These types of information-sharing behaviors are influenced by their promixity to the event. Hughes and Palen [14], in analyzing twitter usage during mass critical events, learnt that information sharing was heightened on the specific day of the event, i.e., hurricane landfall. This demonstrates the contextual importance of information sharing in providing real-time updates that local residents can use to gauge path proximity to their personal property. Relatedly, Twitter has also been used as a tool for risk assessment in these high crisis events. Demuth et al. [10] found that Twitter users from Far Rockaway, New York, engaged with online social and environmental cues to assess their own risk during Hurricane Sandy. The social cues included tweets from peers about local government and business actions, while environment cues included tweets about the natural and built environment. Residents revealed that as the threat grew closer, they relied heavily on these cues to make decisions.

Twitter has also been seen as an avenue to engage in discourse regarding the appropriateness of interventions [9]. Dailey and Starbird [9] found that users turn to each other to make sense of interventions during crisis events. They engage in activities such as assembling evidence that is relevant to local populations and collectively interpret the meaning of specific interventions. Dailey and Starbird [9] also highlight the importance of subject matter experts during such events and note users' reliance on information spread by them.

Beyond the monitoring of usage during critical events, prior work has also advocated for the importance of social media analysis and engagement for effective emergency and policy response. Palen et al. [24] argue that social media engagement can be used to identify needs of members of the public. They note that the public should be seen as "a powerful, self-organizing, and collectively intelligent force, information and communication technology can play a transformational role in crisis"[24, p. 1]. Starbird et al. [34] further augment this by noting that citizen reporting should be viewed by emergency teams as a complementary effort to their official communications.

Our research further supports and augments these findings by engaging with some of them within a Ugandan context. Specifically, we present the ways in which Ugandan Twitter users relied on real-time contextual information about COVID-19 and examine instances of discourse with particular relevance to local health and disaster-preparedness policy makers.

\subsection{Social Media use during disease outbreaks}

Disease surveillance and prediction is not a new phenomenon $[27,29]$; however, the explicit use of social media to do so is a growing field. This, in part, might be attributed to previous fail cases, in which erroneous predictions were made [18]. Despite this, researchers have been successful in building systems to augment self-reported information with Twitter data. In their system called The Flu Outlook platform, Zeng et al. [44] leveraged Twitter data to infer initial infections. This system has been used to forecast the seasonal Influenza in several countries. Similarly, Samira et al. [43] designed a framework that monitored the Avian Influenza in real time. They found that their system can enhance traditional systems by providing early detection of outbreaks. This information is particularly useful to policy makers handling crisis management. These findings are further supported by Signorini et al. [31], who designed a system that used Twitter data to track the prevalence of the Swine Flu. This past literature underscores the importance of Twitter data in mapping disease progression. We contribute to this 
scholarship by elevating the importance of Twitter as source of understanding user conversations around official events and policies during the COVID-19 pandemic. This information is particularly important to policy strategists in health because they can use it to improve on existing awareness and prevention strategies.

The use of Twitter data during crisis events that create vulnerable situations also raises ethical concerns about privacy. Blose et al. [3] found that during the COVID-19 pandemic, there was a heightened level of self-disclosure among users. They attributed this to changes in the emotional state and personal experience, with specific regard to the sudden isolation that the pandemic brought. This work supports the importance of protecting user privacy. As such, the work presented in this paper intentionally omits any personally identifiable attributes such as user ID or username in the presentation of our findings.

\subsection{The influence of heuristics on Twitter}

Research has shown that users of Twitter evaluate information credibility solely based on the content shared [21]. This is in contrast to other research $[25,41]$ that showed evidence of the importance of specific heuristics (e.g., author characteristics) on user engagement. Specifically, Park et al. [25] argue that Twitter users assign credibility not only based on the content but also on cues, such as the number of followers and author reputation. They further state that this credibility is augmented by the Tweet authors' proximity to the event being reported. This work contributes to this conversation by examining and shedding light on key heuristics used and ignored by Ugandan Twitter users to engage during the COVID-19 pandemic.

\section{METHODOLOGY}

The goal of this study is to examine Ugandans' experiences, conversations and engagement patterns on Twitter using tweets that were shared before, during and after 15 nationally televised presidential addresses (from March 18th - June 1st, 2020). We used a mixed-methods approach [13] where both quantitative and qualitative methods were concurrently used to analyze and interpret the twitter dataset on COVID-19 in Uganda. We elaborate on our data collection and analysis approaches in Section 3.1 and 3.3, respectively.

The study was approved by the Clemson University and Carnegie Mellon University Institutional Review Boards (IRB). The tweets analyzed in this study are fully available in the public domain and were accessed without any violation of Twitter's terms of use.

\subsection{Data Collection}

The Twitter data collected and used in this study was obtained from public tweet IDs in the COVID-19 Twitter dataset provided by Chen et al. [7]. The Chen et al. dataset is an ongoing global collection of public Tweet IDs dating back to January 21st, 2020. These Tweet IDs are associated with keywords about COVID-19. This dataset is collected by leveraging Twitter's streaming application programming interface (API) and Tweepy to enable researchers to study "online conversation dynamics in the context of a planetary-scale epidemic outbreak of unprecedented proportions and implications" [7].
To scope our data collection, we focused on Tweets broadcast between March 18th, 2020, and June 1st, 2020. These dates coincided with 15 of the Ugandan president's national addresses on COVID-19. These were the total number of addresses at the time of data collection. We collected a total of 14,791,368 global Tweet IDs associated with these dates. For each national address, we noted the publicised start time information, the actual address start time information, and the entire duration of the session. From this information, we were able to determine the time windows within which to filter our tweet dataset. We set our time parameters for each specific address to start one hour before the publicised address time and end 24 hours later (see Appendix A, Table 3). We converted East African timezone (EAT) to the UTC that is used in the Chen et al. dataset (these were reconverted to East African time during analysis). Once all of the Tweet IDs within the relevant time-frame were collected, we used DocNow's Hydrate tool [36] to collect the content and metadata associated with all of the TweetIDS. We then used Python and Jupyter Notebook to filter the hydrated tweets using eight context specific keywords (uganda, kampala, covid19ug, uganda, uot, staysafeug, m7address, and museveniaddress). This set of keywords includes hashtags that were commonly used during the address, location-specific hashtags, and those used to refer to Ugandans online. As a result, our final dataset had a total of 28,587 tweets.

\subsection{Data Prepossessing}

We cleaned and prepared our data for analysis by removing nonEnglish tweets, mentions, URLS, hashtags, 'RT', usernames, hyperlinks, emoticons, whitespace and punctuation within the tweet text [32].

As with many phenomena on the internet and in the real world, the positive continuous variable data on the number of followers and user-engagement (i.e., retweet counts) within our dataset was highly skewed and not normally distributed. For analysis, we normalized the variables using log-transformation [17] in order to reduce the impact of outliers (i.e., some user(s) having an extremely high number of followers/retweets relative to the majority of other users in our dataset).

\subsection{Data Analysis}

3.3.1 Topic Clustering: We investigated the word clusters that permeated the conversations within the tweets contained in our dataset. Clustering analysis is a technique used to group similar objects in a community together [33]. Thus, we used the Louvian clustering algorithm [2] that identifies communities in large networks to detect the prevalent clusters within the text-based tweets. We ended up with five clusters that we labelled and defined as:

(1) Coronavirus - tweets on the spread and progression of coronavirus.

(2) First Case - tweets on the first COVID-19 case in Uganda.

(3) Presidential Address - tweets about the presidential address.

(4) Lockdown - tweets about the lockdown and stay-at-home orders.

(5) Truck Drivers - tweets about the prevalent cases among truck drivers. 
The common keywords uncovered under each cluster are shown in Table 1 (see Appendix B: Figure 3, for the representative network representation).

3.3.2 Sentiment Analysis: We were also interested in examining the sentiment of the tweets in each cluster. We implemented Hutto and Gilbert's VADER Analysis [12] on original tweets within our database. VADER is a sentiment lexicon that was created and validated using qualitative and quantitative methods and is specifically designed for online content. It uses heuristics (i.e., rules that embody grammatical and syntactical conventions when expressing sentiment) to improve accuracy across multiple online domains (e.g., online blogs, reviews, and social media sites). When compared against individual human raters, VADER boasts of a higher accuracy $(\mathrm{F} 1=0.96$ vs $\mathrm{F} 1=0.84)$ in classifying tweet sentiment.

3.3.3 Statistical Regression model: We conducted a series of linear mixed effects regression models (lmer) to assess and examine whether the twitter heuristics, verification status (verified/unverified) and/or the number of followers of an account had a direct effect on user engagement (i.e., retweet count). We first created a baseline model comprised of a random intercept to account for the within-subjects (i.e., multiple tweets from the same user(s)) markup of our dataset. Next, we added the verification status (verified vs. unverified) and number of followers of an account as additional variables to the baseline model. Using a $\chi^{2}$-based ANOVA comparison, we tested whether there was a significant improvement in model fit upon adding each of these variables to the baseline model. As shown in Table 2, the verification status and number of followers of an account both had pronounced significant effects on user engagement $(\mathrm{p}<.0001)$. The two-way interaction between verification status and number of user followers was also significant $(\mathrm{p}=.0129)$, showing that their effects are interrelated. Furthermore, we conducted post-hoc tests to determine how exactly the verification status and number of followers affected user engagement by conducting separate multilevel models on the 'verified' and 'unverified' accounts. The models specified were the same as the main model but excluded the main effect and interaction term involving the account verification status. The findings are discussed in Section 4.1.2.

3.3.4 Coding and Thematic Analysis. Lastly, we were interested in further examining each topic cluster to reveal the sub-themes (types of tweets) that dominated the user conversations on Twitter. We randomly sampled tweets from each topic cluster $(\mathrm{N}=427)$ and applied thematic analysis techniques [4]. Each of the tweets were coded by two of the authors. They used line-by-line coding and a spreadsheet for the process. The spreadsheet contained five sheets based on the tweets under each cluster, with a column that contained the tweet text. Throughout the coding process, the coders separately memo-ed and constantly compared the tweets to enable them to become familiar and find similarities and differences between the text tweets. In the early rounds of developing the code book, the coders used language from the tweet text to assign initial codes (i.e., In Vivo coding [19]). Herein, not only was the text used to come up with the initial codes, but the meaning of the tweet was also analyzed to classify each tweet into the correct category. Each new code that emerged was given a definition with specified inclusion and exclusion criteria [15]. Coders met weekly after each

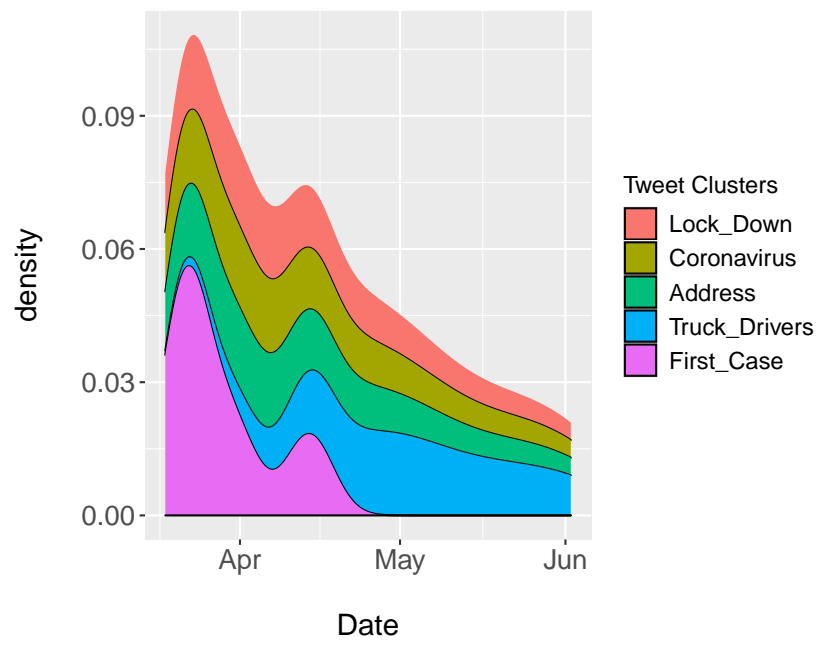

Figure 1: The general distribution of the tweets (coded by topic) in our dataset between March 18th - June, 1st 2020

round to compare memos, definitions and applications of codes, discrepancies, and refinements of the evolving code book. After three rounds, no new themes emerged and the coders had achieved an acceptable level of inter-rater reliability (Cohen's Kappa) [8] of 0.77 . A Cohen's kappa above 0.6 is usually considered satisfactory [20]. The findings are discussed in Section 4.2.

\section{FINDINGS}

\subsection{Quantitative Results}

4.1.1 Topic Clusters and Sentiment: We find that the prevalent tweet topics in our dataset were about the lockdown, coronavirus, presidential address, truck drivers and the first COVID-19 case. Figure 1 shows the emergence and decline patterns of these topics on Twitter over time. We observed a gradual decline in the volume of tweets over time. However, closer inspection revealed a surge around the moments when drastic directives, such as the initial 21-day lockdown and subsequent extension, were issued.

Sentiment analysis of these tweets revealed that similar to the findings of Dubey [11], $42.5 \%$ of the tweets at the time were positive. This suggests that Ugandans took a positive and hopeful approach in the early fight against COVID-19.

4.1.2 Effect of heuristics (account verification status and number of followers) on user engagement: We find that the number of followers of an account significantly predicted user engagement $(b=0.21$, $\mathrm{t}(1122)=5.49, \mathrm{p}<.0001)^{3}$ with a tweet, while verification status $\operatorname{did} \operatorname{not}(\mathrm{b}=0.36, \mathrm{t}(2479)=0.79, \mathrm{p}=.428)$. However, there was a significant interaction effect of both the verification status and number of followers $(b=-0.097, t(1122)=-2.49, p<.05)$, on user engagement.

${ }^{3} \mathrm{~b}$ : the standardized coefficient, t: the calculated difference represented in units of standard error (i.e., the closer $\mathrm{T}$ is to 0 , the more likely there isn't a significant difference), $\mathrm{p}$ : the p-value (probability value) is a probability measure of finding the observed, or more extreme, results, when the null hypothesis of a given statistical test is true (the level of significance of 0.05 is considered conventional and the most commonly used). 
Table 1: Examples of common keywords that were in each of the five cluster topics.

\begin{tabular}{|c|l|c|}
\hline Topic & Keyword & Label \\
\hline 1 & coronavirus, psa, pandemic, uganda, positive, tested, covid & Coronavirus \\
\hline 2 & new, covid, confirmed, first, cases, flight, case, uganda, died, confirms & First Case \\
\hline 3 & today, may, address, pm, country, ug & Presidential Address \\
\hline 4 & total, number, lockdown, lift, day, days, another & Lockdown \\
\hline 5 & truck, samples, tested, positive, negative, drivers & Truck Drivers \\
\hline
\end{tabular}

Table 2: Direct main and interaction effects of verification status and number of followers of an account on User Engagement (significant effects are boldfaced).

\begin{tabular}{l|r|r|r}
\hline Model & $\chi^{2}$ & \multicolumn{1}{|c|}{$d f$} & \multicolumn{1}{c}{$p$-value } \\
\hline retweet_count $\sim(1 \mid$ username $)$ & & & \\
+verification_status & 268.28 & 1 & $<\mathbf{. 0 0 0 1}$ \\
+followers & 370.67 & 2 & $<. \mathbf{0 0 0 1}$ \\
\hline Two way Interactions & & & \\
\hline +verification_status ${ }^{\star}$ followers & 6.18 & 3 & .0129
\end{tabular}

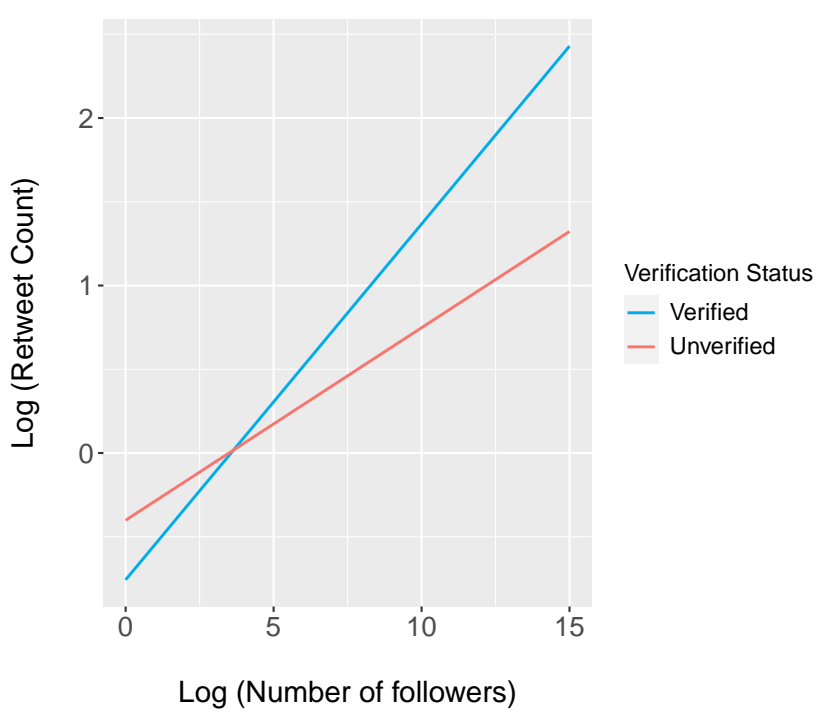

Figure 2: Predicted Effect of the Number of Account Followers on User Engagement, moderated by the Verification Status.

Post-hoc analysis of the interaction effect revealed that the number of followers significantly predicted user engagement for both verified $(\mathrm{b}=0.21, \mathrm{t}(131)=-2.49, \mathrm{p}<.05)$ and unverified accounts $(\mathrm{b}$ $=-0.097, \mathrm{t}(1122)=-2.49, \mathrm{p}<.05)$, but at different levels (see Figure 2 ). User engagement was more pronounced for verified accounts than unverified accounts, which suggests that verification was a key heuristic for increased user engagement on Twitter.

\subsection{Qualitative Results}

In this section, we synthesize the Ugandan Twitter users' conversations embedded within the five clusters and present the high-level themes related to each cluster.

4.2.1 Address Cluster. The Address cluster specifically tagged and highlighted behaviors and sub-themes related to the address.

Twitter users turned citizen pseudo-journalists. During the different addresses, users used their accounts to report their content. The reporting took two distinct forms. In one, users reported on the addresses verbatim. They diligently shared the address to their followers. This style of using Twitter was popular across both journalists (as denoted in their profile descriptions) and regular citizens. In the second category, users resorted to paraphrasing the content, often adding their own commentary to what they felt was a key aspect of the address.

"In short, you're still in lockdown till 2nd fune, 2020. \#m7address"

The amplification of Presidential orders. Twitter provided one platform in which presidential directives were shared in real time. Users also reiterated and amplified new measures and directives that were announced during the course of the address. These directives included actions/orders that were to be effected immediately.

"Mandatory testing of interstate drivers begins today
following president Museveni's address about the issue.
One person yesterday from Kenya tested positive for
corona virus bringing the total to 55 cases..."

A source for real-time schedule updates. As is popular among Twitter users during crises events [40], Ugandan Twitter users also took time to share local contextually relevant information, e.g., regarding schedules of the presidential addresses. These tweets included real-time updates in case of address time changes, delayed starts or postponement as well as platforms on which the event would be broadcast live. Interestingly, several traditional media houses used this as a marketing opportunity to edge out their competition by advertising when they would be broadcasting the address.

"... will broadcast you this address live on at 8.00p.m today. Tune in, you cannot afford to miss"

4.2.2 Lockdown Cluster. As part of the safeguards against the virus, Uganda joined other parts of the world by instituting a lockdown period. This subject soon characterised messaging in both the presidential addresses and conversations around the different addresses. 
Using humor as a fading silver lining. Initial reactions to the lockdown ranged from denoting pessimism around the progression of virus within the country to humor, with some renaming citizens to "Lockdownians". Additionally, sarcasm was embraced as a way to cope with life under the lockdown. For example, when a local telecommunications provider experienced a disruption in its network, a user asked whether the poor service was because their networks were also under lockdown.

"... looks like the... network has been put on lockdown too. So terrible.... Who needs this during this time?...”

As the initial lockdown period began to get extended, tweets quickly began to lose their humorous undertones, replaced with a tone that expressed frustration.

“... seriously, another 14 days! when does it end? the officials don't appear to have a clear strategy, other than the lockdown, despite progressive achievements against covid-19...”

The interruptions of traditions, systems, and lifestyles. The lockdown and its subsequent extension also brought changes in the personal lives of Ugandans, as evidenced by the tweets. Lifestyle changes or interruptions included the increased consumption of Internet data, social isolation, and disruption of family traditions such as travelling to hometowns (villages) for Easter celebrations as well as impacts within the informal sector such as jobs like street vendors, fresh produce stalls in markets, informal public transportation, etc.

Particularly interesting was the emergence of bicycles as one of the safest forms of transportation. In the country's history, bicycles have not been a preferred mode of transportation. Cyclists have often faced discrimination on the road from other motorists. Advocates for sustainable cities used the lockdown as an opportunity to explain the importance of using such clean forms of transportation.

"... cycling is not a curse in lockdown, look at the opportunities for more sustainable cities...”

The tweets also characterised users weighing the pros and cons of being in lockdown. For example, some of these tweets were laced with self-deprecating humor about weight gain and others with fear about the long-term effects of an extended lockdown.

“... I'm either coming out of this lockdown 20lbs lighter, chakras balanced and a house full of completed craft projects or...20lbs heavier with a eating problem..."

4.2.3 First Case Cluster. On March 22nd 2020, Uganda announced the first confirmed case of coronavirus in the country. This cluster particularly documents the different reactions towards this confirmation and news.

The announcement of the local "patient zero". Users propagated information shared from official public sources such as the Ministry of Health as soon as the first case was confirmed in the country. These messages simply reiterated the announcement regarding the confirmation. Additionally, news about the confirmation was augmented with specific information regarding the age, country of travel origin, nationality and gender of this individual. The name of patient zero was kept confidential to safeguard the privacy of the patient.
A call to arms against the virus. The announcement of the first case was also a catalyst used to reinforce earlier safety measures and guidelines. Through the use of motivational language, users took to Twitter to encourage each other to adhere to the official safety guidelines that were instituted by the local official health authority.

“... with Uganda reporting its first case of corona virus infection on 22.03.2020. It is important that we all stay vigilant and safe during \#covid19ug, Ministry of Health has shared directives on how to best prevent and manage infection in Uganda. Please follow these guidelines...”

4.2.4 Coronavirus Cluster. With coronavirus finally manifesting within the country, this cluster of tweets highlights users tracking news about outbreaks both at home and abroad.

Carefully tracking the progression of COVID-19. The tweets revealed that users kept tabs on the development of the disease, both domestically and abroad. Tweets regarding local progression rates highlighted the specific details about the total number of people tested, as well as the daily positive and negative cases. Tweets discussing cases abroad were more generic. They reiterated and discussed increasing case counts, comparisons of local prevention measures with neighboring countries as well as fatality announcements.

Embracing all aspects of hope in the midst of a pandemic. Tweets denoted a reliance on official guidelines and safety measures to mitigate the spread of the virus within the country. Users took to encouraging each other as well as reiterating the officially announced safeguards. Similar to a previous cluster, motivational language was used to remind others in the community that the country had seen worse and as such would get through this particular pandemic.

“... Uganda goes to war with an invisible enemy, we have faced very many situations in our country and I believe as we fight adversity we can win the war against the coronavirus, hold the Ugandan flag high as we all stand united and fight together for our country, may god bless Uganda..."

While sharing about the different impacts of the pandemic (e.g., social, judicial, and economic), users also noted that the pandemic has created space for opportunities of self-care and new businesses.

"... the lighter side of this whole \#coronavirus craziness for \#mentalhealth wellness fanatics is that we get to have ample time for some \#selfcare and \#selflove activities without being in a rush to fly out the door. so, we actually should not be that mad to be \#socialdistancing...”

Relatedly, users also resorted to the use of religion as a way to cope with the impact and effect of the coronavirus pandemic. These tweets made specific reference to religious scriptures.

"... how the armor of God is meant for crises like coronavirus..."

Fact vs. fiction about COVID-19. Instances of misinformation were observed within this cluster. Specifically, this included the notion that race had an influence on whether people got infected with the virus. This particular idea was common in the initial days of 
reporting about the virus. Additionally, there were unsubstantiated claims about different cures of COVID-19.

$$
\text { “... black people don't die from coronavirus ...” }
$$

4.2.5 Truck Drivers Cluster. Uganda is a landlocked country, and thus, there were concerns about the continued operation of truck drivers amidst the lockdown. Truck drivers, who are essential workers [1], ferry goods into the country or further inland to countries like South Sudan, Rwanda, and Congo. Within the context of Twitter discussions, concerns mounted because the truck drivers were driving in from countries that had higher case counts than Uganda.

Public health vs trade. After lockdown was instituted in the country, users noted that truck drivers still had free rein to enter and leave the country. They expressed opinions laced with fear about the possible spread of COVID-19 in relation to the free movement of truck drivers.

"... as long as we have food, the lockdown can go on. However, we expect something to be done about the truck drivers, Mr. M7, please we don't need u to make suggestions, you're the president just state it, "truck drivers should carry their food $n$ not talk to anyone $n$ it shall be done..."

In an effort to counter new potential spreaders and quell the growing negative sentiment among citizens, the government implemented new procedures regarding truck drivers. Directives within the presidential address that specifically targeted these drivers were further propagated within the community.

“... president says they initially allowed truck drivers to continue with their journeys without covid-19 results because they wanted to remove the congestion at the border and minimise costs for drivers and the truck drivers. But now no driver will proceed minus their results ..."

Relatedly, there was mention of retaliation of truck drivers to the policies that were implemented (e.g., staging protests at the border).

Disease progression amidst a specific population. Previously, users kept track of the number of general cases within the country. However, after reports of truck drivers with infections circulated, users and public health officials resorted to separately tracking the infection rates among truck drivers.

“... Uganda now has 79 total confirmed cases with 52 recoveries, 13 active cases, no deaths. The truck drivers were sent to their countries; 6 Kenyans and 8 Tanzanians..."

\section{DISCUSSION}

Our findings provide insight into the topics, conversations, and engagement of Ugandans on Twitter during the pandemic. In this section, we distill these findings and highlight the main ways in which Twitter was used. Overall, the discussion informs on the effective usage of social media during crises (e.g., pandemics, epidemics, landslides, etc.) within developing countries.

\subsection{The Usage of Twitter in a Pandemic}

Based on topic analysis and examination of the conversations, we find that Twitter was a relevant social media platform that was primarily used to communicate, inform and offer support to the public at the onset of the pandemic in Uganda. Below, we describe the main ways through which Twitter was used.

5.1.1 Twitter as a Communication Channel: We find that Twitter was an essential communication channel used to convey, amplify and share information pertaining to the safeguards against COVID19 , active and recovered case counts, the presidential address agenda and key directives to follow in the fight against the pandemic in Uganda. Specifically, Twitter was an avenue through which users were able to react and share the key aspects of the presidential address in real time via their personal accounts. This allowed them to reflect, express and inform the responsible government/public health authorities of the issues that they felt needed to be addressed, such as their displeasure with the policy that allowed truck drivers to continue to operate whilst under the lockdown. Twitter also offered government authorities the opportunity to respond and sensitize users on the underlying rationale of the directives issued. It is possible, therefore, that through a combination of traditional (e.g., radio, television) and Twitter (social media), the communication between the government and the public was bidirectional and beneficial to both parties. We therefore advocate for the continued use and integration of social media as an additional communication component in a government's crisis-response strategic plan.

5.1.2 Twitter as an Information Channel: Our findings also confirm prior work by Sophie et al. [16] that finds Twitter is a good source of information for public health : we find that people used Twitter to get information and updates on the progression of COVID-19. For example, information about the first COVID-19 case in Uganda was highly propagated on the platform. However, we notice that people tried to personally identify the patient beyond the information that was provided. Therefore, we suggest that in the provision of information especially about patients and/or disaster victims, their privacy be safeguarded [23] to protect them from identification and stigma from society. Additionally, we find instances of misinformation about COVID-19 such as purported cures. We recommend that social media platforms design and display informational hubs that contain factual information front and center on the platform as well as take down or place labels on any misinforming content.

5.1.3 Twitter as a Support Channel: Relatedly, we find that people used Twitter as a place to seek and offer support during the pandemic. We observe the surge in the volume of tweets at the onset of the pandemic, use of humorous tweets, and tweets detailing people's lifestyle changes and experiences due to the pandemic. This suggests that Twitter was a platform where people came to share their experiences and seek support from others, especially at the onset of lockdown and stay-at-home orders when most people were physically apart. Overall, the observed interpersonal tweets also indicate that people bonded around the topic of COVID-19 on Twitter. 


\subsection{The Power Of Verification (}

We find that Ugandans engaged more with tweets from verified accounts relative to non-verified accounts. Verified accounts are normally reserved for accounts of public interest and are indicated with a badge ( next to the account username to let people know that the account is authentic [38]. These accounts, on average, have far more followers than non-verified accounts and thus have a greater opportunity for user engagement (e.g., retweets and likes) [38]. However, we find that user engagement of these accounts was not significantly predicted by the number of followers; therefore, there is an additional impact of verification. These findings suggest that Ugandans mostly engaged with tweets from credible sources and support Morris et al.'s [21] findings that an account's verification status on Twitter is an important heuristic that users weigh when evaluating or engaging with information on their timeline, especially during crises such as a pandemic. These findings also reflect the desire of citizens to have accurate updates about the status of the pandemic that they were able to get through these accounts, confirming previous findings [16].

We also observed specific communication strategies that verified users in Uganda used to convey information to the public, such as providing factual information about the contents of the presidential addresses, lockdown, infection counts, and other important news. These strategies reflected those described in previous research [39]. In times of crises, government and news media employees, "as information providers through their personal social media accounts, yield stronger positive effects from the public - as they are viewed as credible sources of information " [39, p. 37]. This form of communication and outreach strategy engages the general public and reassures them during crises [28].

Although verified accounts had a particularly strong impact among Ugandans on Twitter, we did find that non-verified accounts could also have significant impacts, particularly when they had a high number of followers. With more followers, there was a greater chance of engagement.

Overall, we recommend that public health information and awareness campaigns are communicated via verified social media accounts, as we observe that users are more likely to engage and consider verified accounts credible sources of information, especially during times of crises such as a pandemic. We also suggest that authentic government/public health authorities apply for verification on Twitter if they are yet to be verified because they are of high public interest.

\section{LIMITATIONS}

Our data came from only one social media platform, Twitter. While Twitter is one of the largest social media sites in use today, and therefore we feel it is a representative platform for conducting our work, we can only hypothesize about the generalizability of our findings to other platforms. Similarly, the data were comprised of tweets from individuals who had access to and use Twitter with public profiles, thereby limiting generalizability to those who do not use Twitter or have private accounts. Additionally, we used retweet counts as a measure for user engagement to learn about the dissemination of information about COVID-19, but there are other forms of user engagement on Twitter such as favorites, comments, mentions and link clicks.

In the retrieval of the relevant tweets, we used a list of keywords and focused on English tweets broadcast between March 18 and June 1st, 2020, centered around the Ugandan president's 15 national addresses. This might have resulted in a selection bias: many tweets might have been relevant but did not mention the predefined keyword or were out of the selection parameters we used, and those that did include the keyword may still have been irrelevant. As is common with qualitative analyses, there may have been coder bias that influenced our qualitative coding: to mitigate this, we intentionally double coded and reached a high inter-rater agreement. Additionally, we coded only 457 of 28,587 pieces of tweet content; while these tweets were randomly sampled, it is possible that the tweets that we coded were not representative and thus resulted in biased findings. Finally, the tweets that we analysed were mostly in English and thus are not representative of all the tweets in our dataset.

\section{CONCLUSION}

In this paper, we explore and present Ugandans' discourse, experiences and engagement patterns on Twitter around moments leading to, during, and after the Ugandan national presidential addresses regarding COVID-19. We find that tweets from verified accounts had more engagement (i.e., retweets) than tweets from non-verified accounts and suggest that government/public health authorities apply for verification if they seek more user engagement and wide reach of their messages on social media, especially during crises. We also find that most of the tweets at the onset of the pandemic in Uganda were about the following topics: 1) the spread and progression of coronavirus, 2) the first COVID-19 case in Uganda, 3) the presidential addresses, 4) the lockdown directives (i.e., stay-at-home orders), and 5) the prevalent cases among truck drivers who were granted permission to continue operating across the borders during the lockdown. In summary, we provide insight in the specific usage of Twitter as a communication, information and support channel during a pandemic. These findings could be applied to improve the use of social media for communication, awareness, information, and support during crises such as a pandemic.

\section{ACKNOWLEDGMENTS}

We thank Chen et al. [7], whose dataset enabled us collect the Twitter data that was used in this study. We also thank the Mechanism Design for Social Good (MD4SG) Development working group for the engaging discussions that contributed to this work. We also thank Elizabeth J Carter for all the feedback. Lastly, we thank our reviewers for their insightful comments. Moses Namara acknowledges support from a Facebook Fellowship.

\section{REFERENCES}

[1] Nicholas Bariyo. 2020. On East Africa's Borders, Vast fams as Truckers Are Tested for Covid-19. Wall Street Journal. Retrieved 28 September 2020 from https://www.wsj.com/articles/at-africas-borders-vast-jams-as-truckersare-tested-for-covid-19-11591963201

[2] Vincent D Blondel, Jean-Loup Guillaume, Renaud Lambiotte, and Etienne Lefebvre. 2008. Fast Unfolding of Communities in Large Networks. Journal of statistical mechanics: theory and experiment 2008, 10 (2008), P10008. 
[3] Taylor Blose, Prasanna Umar, Anna Squicciarini, and Sarah Rajtmajer. 2020 Privacy in Crisis: A Study of Self-disclosure During the Coronavirus Pandemic. arXiv. arXiv:2004.09717

[4] Virginia Braun and Victoria Clarke. 2006. Using Thematic Analysis in Psychology. Qualitative research in psychology 3, 2 (2006), 77-101. https://doi.org/10.1191/ 1478088706qp063oa

[5] Samantha Dawn Breslin, Thyge Ryom Enggaard, Anders Blok, Tobias Gårdhus, and Morten Axel Pedersen. 2020. How We Tweet About Coronavirus, and Why: A Computational Anthropological Mapping of Political Attention on Danish Twitter during the COVID-19 Pandemic. Somatosphere: Science, Medicine, and Anthropology. Retrieved 22 January 2021 from http://somatosphere.net/forumpost/covid19danish-twitter-computational-map/

[6] Lauren E Charles-Smith, Tera L Reynolds, Mark A Cameron, Mike Conway, Eric HY Lau, Jennifer M Olsen, Julie A Pavlin, Mika Shigematsu, Laura C Streichert, Katie J Suda, et al. 2015. Using Social Media for Actionable Disease Surveillance and Outbreak Management: A Systematic Literature Review. PloS one 10, 10 (2015), e0139701.

[7] Emily Chen, Kristina Lerman, and Emilio Ferrara. 2020. Tracking Social Media Discourse About the COVID-19 Pandemic: Development of a Public Coronavirus Twitter Data Set. FMIR Public Health and Surveillance 6, 2 (2020), e19273.

[8] Jacob Cohen. 1960. A Coefficient of Agreement for Nominal Scales. Educational and psychological measurement 20, 1 (1960), 37-46.

[9] Dharma Dailey and Kate Starbird. 2015. "It's Raining Dispersants": Collective Sensemaking of Complex Information in Crisis Contexts. In Proceedings of the 18th ACM Conference Companion on Computer Supported Cooperative Work \& Social Computing (Vancouver, BC, Canada) (CSCW'15 Companion). Association for Computing Machinery, New York, NY, USA, 155-158. https://doi.org/10. 1145/2685553.2698995

[10] Julie L Demuth, Rebecca E Morss, Leysia Palen, Kenneth M Anderson, Jennings Anderson, Marina Kogan, Kevin Stowe, Melissa Bica, Heather Lazrus, Olga Wilhelmi, et al. 2018. "Sometimes da\# beachlife ain't always da wave": Understanding People's Evolving Hurricane Risk Communication, Risk Assessments, and Responses using Twitter Narratives. Weather, climate, and society 10, 3 (2018), 537-560.

[11] Akash Dutt Dubey. 2020. Twitter Sentiment Analysis during COVID19 Outbreak SSRN. Retrieved 22 January 2021 from http://dx.doi.org/10.2139/ssrn.3572023

[12] Che Gilbert and Erric Hutto. 2014. Vader: A Parsimonious Rule-based Model for Sentiment Analysis of Social Media Text. In Eighth International Conference on Weblogs and Social Media (ICWSM-14), Vol. 81. The AAAI Press, Palo Alto, California, 82. http://comp.social.gatech.edu/papers/icwsm14.vader.hutto.pdf

[13] William E Hanson, John W Creswell, Vicki L Plano Clark, Kelly S Petska, and J David Creswell. 2005. Mixed Methods Research Designs in Counseling Psychology. Fournal of counseling psychology 52, 2 (2005), 224.

[14] Amanda Lee Hughes and Leysia Palen. 2009. Twitter Adoption and Use in Mass Convergence and Emergency Events. International journal of emergency management 6, 3-4 (2009), 248-260.

[15] Staci Neas Johnson. 2019. Cognitive Processes in Undergraduate Anatomy and Physiology Courses. Ph.D. Dissertation. Clemson University, Clemson, SC, USA.

[16] Sophie E Jordan, Sierra E Hovet, Isaac Chun-Hai Fung, Hai Liang, King-Wa Fu, and Zion Tsz Ho Tse. 2019. Using Twitter for Public Health Surveillance from Monitoring and Prediction to Public Response. Data 4, 1 (2019), 6.

[17] Oliver N Keene. 1995. The Log Transformation is Special. Statistics in medicine 14, 8 (1995), 811-819.

[18] David Lazer, Ryan Kennedy, Gary King, and Alessandro Vespignani. 2014. The Parable of Google Flu: Traps in Big Data Analysis. Science 343, 6176 (2014) 1203-1205. https://doi.org/10.1126/science.1248506

[19] Jimmie Manning. 2017. In Vivo Coding. The international encyclopedia of communication research methods 1,1 (2017), 1-2. https://doi.org/10.1002/9781118901731. iecrm0270

[20] Mary L McHugh. 2012. Interrater Reliability: The Kappa Statistic. Biochemia medica: Biochemia medica 22, 3 (2012), 276-282.

[21] Meredith Ringel Morris, Scott Counts, Asta Roseway, Aaron Hoff, and Julia Schwarz. 2012. Tweeting is Believing? Understanding Microblog Credibility Perceptions. In Proceedings of the ACM 2012 conference on computer supported cooperative work. Association for Computing Machinery, New York, NY, USA 441-450.

[22] Esther Nakkazi. 2020. Obstacles to COVID-19 Control in East Africa. The Lancet. Infectious Diseases 20, 6 (2020), 660.

[23] Moses Namara, Daricia Wilkinson, Byron M Lowens, Bart P Knijnenburg, Rit Orji, and Remy L Sekou. 2018. Cross-Cultural Perspectives on eHealth Privacy in Africa. In Proceedings of the Second African Conference for Human Computer Interaction: Thriving Communities. ACM, New York, US, 1-11.

[24] Leysia Palen, Kenneth M. Anderson, Gloria Mark, James Martin, Douglas Sicker, Martha Palmer, and Dirk Grunwald. 2010. A Vision for Technology-Mediated Support for Public Participation \& Assistance in Mass Emergencies \& Disasters. In Proceedings of the 2010 ACM-BCS Visions of Computer Science Conference (ACM$B C S$ '10). BCS Learning \& Development Ltd., Swindon, GBR, Article 8, 12 pages.
[25] Chang Sup Park and Barbara K Kaye. 2019. Expanding Visibility on Twitter: Author and Message Characteristics and Retweeting. Social Media+ Society 5, 2 (2019), 2056305119834595.

[26] Han Woo Park, Sejung Park, and Miyoung Chong. 2020. Conversations and Medical News Frames on Twitter: Infodemiological Study on COVID-19 in South Korea. Journal of Medical Internet Research 22, 5 (2020), e18897.

[27] Leo LM Poon, Timothy Song, Roni Rosenfeld, Xudong Lin, Matthew B Rogers, Bin Zhou, Robert Sebra, Rebecca A Halpin, Yi Guan, Alan Twaddle, et al. 2016. Quantifying influenza Virus Diversity and Transmission in Humans. Nature genetics 48, 2 (2016), 195-200.

[28] H Raghav Rao, Naga Vemprala, Patricia Akello, and Rohit Valecha. 2020. Retweets of Officials' Alarming vs Reassuring Messages During the COVID-19 Pandemic: Implications for Crisis Management. International fournal of Information Management 3 (2020), 102187.

[29] Nicholas G Reich, Logan C Brooks, Spencer J Fox, Sasikiran Kandula, Craig J McGowan, Evan Moore, Dave Osthus, Evan L Ray, Abhinav Tushar, Teresa K Yamana, et al. 2019. A Collaborative Multiyear, Multimodel Assessment of Seasonal Influenza Forecasting in the United States. Proceedings of the National Academy of Sciences 116, 8 (2019), 3146-3154.

[30] Philip J Rosenthal, Joel G Breman, Abdoulaye A Djimde, Chandy C John, Moses R Kamya, Rose GF Leke, Matshidiso R Moeti, John Nkengasong, and Daniel G Bausch. 2020. COVID-19: Shining the Light on Africa. The American fournal of Tropical Medicine and Hygiene 102, 6 (2020), 1145.

[31] Alessio Signorini, Alberto Maria Segre, and Philip M Polgreen. 2011. The Use of Twitter to Track Levels of Disease Activity and Public Concern in the US During the Influenza A H1N1 Pandemic. PloS one 6, 5 (2011), e19467.

[32] Tajinder Singh and Madhu Kumari. 2016. Role of Text Pre-processing in Twitter Sentiment Analysis. Procedia Computer Science 89, Supplement C (2016), 549-554.

[33] Sandip Sinharay. 2010. An Overview of Statistics in Education. International Encyclopedia of Education 3 (2010), 1-11. https://doi.org/10.1016/B978-0-08044894-7.01719-X

[34] Kate Starbird, Leysia Palen, Amanda L. Hughes, and Sarah Vieweg. 2010. Chatter on the Red: What Hazards Threat Reveals about the Social Life of Microblogged Information. In Proceedings of the 2010 ACM Conference on Computer Supported Cooperative Work (Savannah, Georgia, USA) (CSCW'10). Association for Computing Machinery, New York, NY, USA, 241-250. https: //doi.org/10.1145/1718918.1718965

[35] Yue Su, Jia Xue, Xiaoqian Liu, Peijing Wu, Junxiang Chen, Chen Chen, Tianli Liu, Weigang Gong, and Tingshao Zhu. 2020. Examining the Impact of COVID-19 Lockdown in Wuhan and Lombardy: A Psycholinguistic Analysis on Weibo and Twitter. International fournal of Environmental Research and Public Health 17, 12 (2020), 4552 .

[36] Documenting the Now. 2020. Hydrate Tool [Computer Software]. GitHub. https: //github.com/DocNow/hydrator

[37] Twitter. 2020. About your activity dashboard. https://help.twitter. com/en/managing-your-account/using-the-tweet-activity-dashboard\#: : text=Engagements:Totalnumberoftimes, ofengagementsdividedbyimpressions

[38] Twitter. 2020. Verified account FAQs. https://help.twitter.com/en/managingyour-account/twitter-verified-accounts

[39] Ward van Zoonen and Toni van der Meer. 2015. The Importance of Source and Credibility Perception in Times of Crisis: Crisis Communication in a Socially Mediated Era. Journal of public relations research 27, 5 (2015), 371-388.

[40] Sarah Vieweg and Kate Starbird. 2010. Microblogging in Mass Emergency. Citeseer. Retrieved September 20, 2020 from https://www.academia.edu/13371046/ Microblogging in Mass Emergency

[41] Chunyan Wang and Bernardo A Huberman. 2012. Long Trend Dynamics in Social Media. EPf Data Science 1, 1 (2012), 2.

[42] WHO. 2020. WHO Director-General's Opening Remarks at the Media Briefing on COVID-19 - 11 March 2020. World Health Organization. Retrieved 22 January 2021 from https://www.who.int/dg/speeches/detail/who-director-general-sopening-remarks-at- the-media-briefing-on-covid-19---11-march-2020\#: text=WHOhasbeenassessingthis, touselightlyorcarelessly.

[43] Samira Yousefinaghani, Rozita Dara, Zvonimir Poljak, Theresa M Bernardo, and Shayan Sharif. 2019. The Assessment of Twitter's Potential for Outbreak Detection: Avian Influenza Case Study. Scientific reports 9, 1 (2019), 1-17.

[44] Qian Zhang, Corrado Gioannini, Daniela Paolotti, Nicola Perra, Daniela Perrotta, Marco Quaggiotto, Michele Tizzoni, and Alessandro Vespignani. 2015. Social Data Mining and Seasonal Influenza Forecasts: The Fluoutlook Platform. In foint European Conference on Machine Learning and Knowledge Discovery in Databases. Springer, New York, US, 237-240. 


\section{A ADDRESS TIME PARAMETERS}

Table 3: Example Time parameters for the 8th National Address (Apr 3rd, 2020)

\begin{tabular}{l|l}
\hline Advertised start & 8PM EAT (5PM UTC) \\
Actual start & 8.30PM EAT (5.30PM UTC) \\
Entire session & 1hr 50 mins \\
Tweet IDs collected & Apr 3rd, 4PM - April 4th, 5PM (UTC) \\
\hline
\end{tabular}

journalist television station blog entertainment personality government official newspaper government body un affiliated business personality

\section{B NETWORK REPRESENTATION OF THE FIVE CLUSTERS DISTINGUISHED BY COLOR}

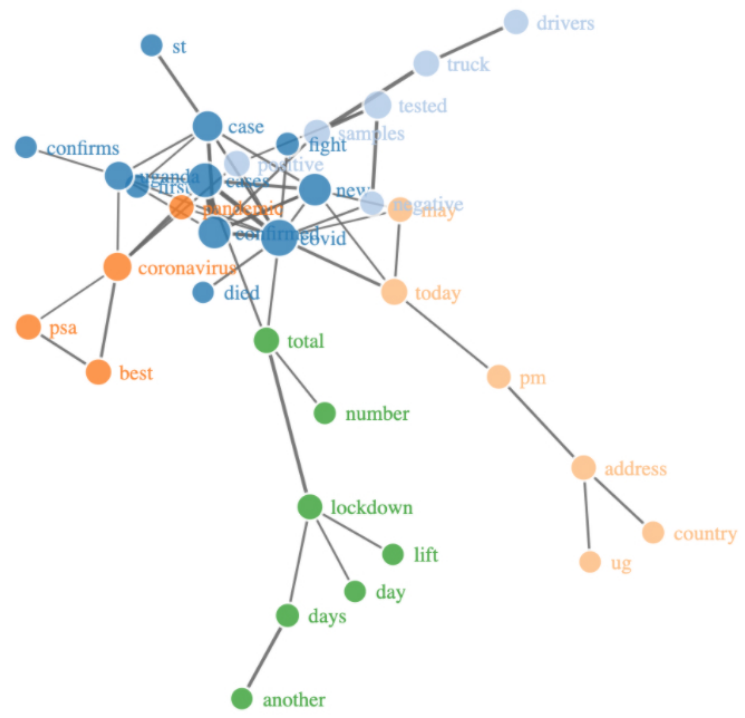

Figure 3: A network representation of the 5 clusters coded by color 